pag

Business School

WORKING PAPER SERIES

Working Paper

$2014-254$
Réussir la diversité du genre. Une expérience de développement de l'égalité professionnelle femme/homme dans le secteur de la construction, souvent considéré comme « masculin » : l'exemple de CARI, entreprise de BTP dans les Alpes Maritimes

Marie José Scotto

Robin Sappe

André Boyer

http://www.ipag.fr/fr/accueil/la-recherche/publications-WP.html

IPAG Business School

184, Boulevard Saint-Germain

75006 Paris

France 


\title{
Réussir la diversité du genre. Une expérience de développement de l'égalité professionnelle femme/homme dans le secteur de la construction, souvent considéré comme « masculin » : l'exemple de CARI, entreprise de BTP dans les Alpes Maritimes
}

\author{
Marie José Scotto \\ IPAG Ecole Supérieure de Commerce, m.scotto.@.ipag.fr \\ Robin Sappe \\ Directeur du développement des ressources humaines et de la diversité chez CARI, Rsappe@cari.fr \\ André Boyer \\ Professeur, CRIFP-IAE de Nice
}

\section{Résumé}

\section{Français}

Cet article traite de la diversité du genre, appréciée en tant qu'élément stratégique de développement des ressources humaines au sein des entreprises. Le terrain de référence est constitué par des métiers et des entreprises, dits masculins. Ces métiers et ses secteurs s'engagent dans une politique de féminisation inscrite dans une logique de développement de l'égalité professionnelle femmes/hommes. La recherche se centre sur les processus qui permettent la mise en ouvre de cette modification de la démographie de la force de travail au sein de l'entreprise, et analyse le cas d'une entreprise de bâtiment. Il ressort de cette approche non seulement un bénéfice d'image dont peut bénéficier tout un secteur, mais également une réflexion plus large sur le modèle de l'organisation.

English

This article deals with gender diversity as a key component of HR strategy. The field of investigation is composed by jobs and companies viewed as typically male. These sectors are tending to develop gender diversity and professional equality between women and men. This current research is based on the processes allowing this change in the demography of the company work force and explores the case of a building contractor company. It becomes evident that this approach brings a real image benefit but initiates as well a wider frame of thought for the organisational model.

Post Print : Scotto Marie José et al., "Réussir la diversité du genre. Une expérience de développement de l'égalité professionnelle femme/homme dans le secteur de la construction, souvent considéré comme "masculin »: l'exemple de CARI, entreprise de BTP dans les Alpes Maritimes », Management \& Avenir 4/ 2008 ( $\left.n^{\circ} 18\right)$, p. 18-41. 
a problématique de cet article s'articule autour de la prise en compte de la diversité du genre dans l'entreprise, l'objectif managérial consistant à en faire un élément stratégique de succès de l'entreprise. Nous présenterons dans une première partie le cadre théorique de notre réflexion. Tout d'abord en analysant les conditions de l'émergence du concept de diversité, nous considérons que cet aspect de la diversité (le genre) est fortement lié aux problématiques de l'égalité professionnelle hommes / femmes dans l'entreprise.

Nous nous intéresserons ensuite, en nous appuyant sur une perspective historique, à la notion de métiers «féminins» et «masculins » et aux stéréotypes qui s'y rattachent. Nous montrerons l'évolution des perceptions liées au travail féminin au gré de l'histoire et des époques en tentant d'éclairer les lignes de continuité ou de rupture et ce plus particulièrement dans le contexte du secteur du Bâtiment et des Travaux Publics (BTP) en France, secteur souvent considéré comme un bastion des métiers dits masculins.

Dans une dernière partie, nous utiliserons le cas de CARI, entreprise nationale de BTP dont le siège se situe dans les Alpes Maritimes. L'analyse de l'expérience réalisée dans cette entreprise permettra de mettre en lumière dans ce cas précis, les processus qui amènent à la mise en œuvre du développement de la diversité du genre, ici la féminisation de métiers dits et perçus comme masculins. Dans un second temps, la discussion portera d'une part sur les difficultés rencontrées sur le terrain mais également sur les apports attendus de la réussite du processus engagé et notamment les changements induits dans les processus managériaux. Notre article s'inscrit dans le cadre d'une thèse en Sciences de gestion sur l'intégration par le management de l'organisation de la diversité des genres.

\section{1 - Le concept de diversité}

\section{- Les conditions d'émergence}

Le concept de diversité trouve sa source aux Etats-Unis, dans les luttes des minorités pour leurs droits civiques avec l'émergence des politiques d'égalité des chances (Equal Employment Opportunities ou EEO) au cours des années soixante. Les objectifs sont clairement de faciliter l'accès de certaines minorités et des femmes aux opportunités d'emploi. Ils conduiront à la mise en place d'actions plus spécifiques et supposées plus efficaces de discrimination positive (Affirmative Action ou AA) concernant notamment les procédures d'embauche et de promotion. (Bender 2004). Ces actions prennent la forme de «traitement préférentiel» envers des groupes spécifiés (Sabbagh 2004), afin de compenser des mesures discriminatoires dont ils auraient pu être victimes auparavant. La remise en cause de cette approche peut être datée aux Etats-Unis de 1974 avec le cas Allan Bake versus Regents of University of California qui conteste le programme d'affirmation action pratiquée 
par l'Université de Californie sous la forme d'un pourcentage de places réservées aux minorités ethniques. Ce cas, particulièrement long et qui ne se conclura qu'en 1978, sera à l'origine d'une approche plus globale de «diversité », au sein de laquelle l'appartenance ethnique est présentée comme une composante parmi d'autres (Sabbagh 2004), qui doit être prise en compte dans un corps d'éléments pluriels. Le concept de diversité constitue donc une approche différente, plus stratégique, mieux à même que le concept d'égalité des chances de lutter contre les discriminations en ménageant les intérêts de l'individu et de l'entreprise (Cox 1991, Thomas et Ely 1996). Car ici se trouve la différence de perspective essentielle : le passage du groupe à l'individu (Bender 2004). Cette logique de différentiation est soulignée par des auteurs tels que Prasad et Cavanaugh qui l'inscrivent dans une dimension stratégique de l'entreprise (Prasad et alii., 2002).

\section{- Les aspects de la diversité}

Ce concept ne se limite pas à l'origine ethnique, mais s'étend afin d'englober de nouvelles composantes comme le sexe, s'articulant ainsi avec les problématiques de l'égalité professionnelle femme/homme, la préférence sexuelle, l'âge et le handicap. Nous sommes là dans un modèle intégratif d'une «main d'œuvre hétérogène » [1] Etude ORSE (2004). L'accès des femmes aux postes de... $[1]$. Nos travaux s'attachent plus spécifiquement à cerner cette diversité du genre, visant à développer l'accession des femmes aux différents niveaux de la hiérarchie de l'entreprise. Il nous semble que la diversité du genre s'inscrit dans un paradigme spécifique par rapport à l'origine du concept de diversité, considérant que les femmes, fortes d'une représentation de plus de $46 \%$ de la population active, peuvent difficilement être considérées comme une minorité. Cette question de la diversité du genre relève d'une transversalité bien particulière. En fait «l'égalité des chances insiste davantage sur l'action corrective et sur la surveillance du nombre de personnes formées, embauchées ou promues dans certains groupes sous-représentés » [2] La promotion des femmes aux postes de décision. 199.... [2], ici les femmes.

La diversité, quant à elle peut se définir comme « un besoin interne de changement destiné à répondre aux besoins économiques et commerciaux internes découlant de la représentativité des femmes dans la population active et du besoin d'utiliser tous les domaines de compétences dans la recherche de la survie et de l'efficacité de l'organisation » [3] Kandola, R. Fullerton, J. (1994). Diversity : more... [3]. Des auteurs comme Anne Françoise Bender et Frédérique Pigeyre soulignent la convergence des deux approches et le mérite des programmes d'égalité professionnelle pour développer la représentation des femmes au niveau de l'encadrement. Les travaux de Kandolla et Fullerton présentent de manière synthétique les principales caractéristiques des deux approches. 


\begin{tabular}{|l|l|}
\hline Gestion de la diversité & Egalité des chances \\
\hline $\begin{array}{l}\text { Permet à tous les salariés de maximiser leur } \\
\text { potentiel et leur contribution à l'organisation }\end{array}$ & $\begin{array}{l}\text { Se concentre sur les questions de } \\
\text { discrimination }\end{array}$ \\
\hline $\begin{array}{l}\text { Englobe un large éventail d'individus : nul n'est } \\
\text { exclu }\end{array}$ & $\begin{array}{l}\text { Se concentre sur les femmes, les minorités } \\
\text { ethniques et les personnes handicapées }\end{array}$ \\
\hline $\begin{array}{l}\text { Se concentre sur le mouvement au sein de } \\
\text { l'organisation et de la réalisation des objectifs } \\
\text { commerciaux }\end{array}$ & $\begin{array}{l}\text { Met moins l'accent sur le changement culturel et } \\
\text { la réalisation des objectifs commerciaux }\end{array}$ \\
\hline $\begin{array}{l}\text { Est la préoccupation de l'ensemble des salariés } \\
\text { et en particulier des cadres }\end{array}$ & $\begin{array}{l}\text { Concerne principalement des spécialistes du } \\
\text { personnel et des ressources humaines }\end{array}$ \\
\hline Ne repose pas sur l'action corrective/positive & Repose sur l'action positive \\
\hline $\begin{array}{l}\text { Source : selon Kandulla } R \text { et Fullerton J, cité par Bender AF in Revue Française de Gestion. Août } \\
2004\end{array}$
\end{tabular}

L'analyse des pratiques d'entreprises qui se développent actuellement en France et en Europe montre que les mesures mises en œuvre notamment dans les pratiques d'égalité professionnelle et de développement de la diversité du genre empruntent en fait aux deux approches (Bender 2004), et surtout, même si la référence n'y est pas explicite, aux théories de «l'affirmative action ».

\section{1 - Un nouveau paradigme accepté par tous?}

La diversité du genre suscite d'autant moins de controverse, qu'elle s'appuie sur la notion de performance de l'organisation. Les auteurs s'accordent tous sur les raisons de promouvoir la diversité au sein des entreprises : la nécessité d'un «sourcing » plus large de la ressource humaine, l'amélioration des performances commerciales afin de refléter la diversité des clients, l'accent mis sur le lien entre diversité des équipes et innovation et créativité, la contrainte des normes nationales et internationales, les attentes plus globales de la société concernant l'entreprise citoyenne (Peretti 2006). Ce besoin de diversité s'articule historiquement autour de deux pôles (Cornet 2002): le marketing et la performance commerciale des entreprises, mais également l'éthique au travers du renouveau du concept de responsabilité sociale des entreprises.

\section{Le « business case » de la diversité du genre}

Nous avons développé dans un précédent article [4] Boyer A, Scotto M.J. (2006). La mixité du genre est... $\lceil 41$, les enjeux pour les entreprises ainsi que les facteurs qui favorisent le développement de la diversité du genre et de l'égalité professionnelle femme/homme dans l'entreprise. Les auteurs ont montré que les actions conjuguées des différentes parties prenantes en faveur des pratiques d'égalité professionnelle et de développement de la diversité du genre font prendre conscience aux entreprises des désavantages à ne pas se saisir de ce problème. Les risques pour l'organisation sont évalués en terme d'image et de réputation, mais également en terme 
de modification de l'environnement législatif en France et en Europe. (Boyer. A, Scotto MJ. 2006).

\section{La diversité du genre : une problématique de responsabilité sociale ?}

Au travers des polémiques et des discussions sur la parité femme/homme, ou sur l'intégration professionnelle des femmes, la question de l'égalité des sexes et de l'accession des femmes à tous les niveaux de l'entreprise s'inscrit comme une dimension majeure de la responsabilité sociale des entreprises (de Bry F, Ballet J.2006). Il peut être utile de rappeler le concept de Responsabilité Sociétale de l'Entreprise (RSE), en s'appuyant sur la définition proposée par le livre vert de la Commission Européenne: «intégration volontaire par les entreprises de préoccupations sociales et environnementales à leurs activités commerciales et leurs relations avec leurs parties prenantes ». La Commission Européenne insiste également sur le fait que « être socialement responsable signifie non seulement satisfaire pleinement aux obligations juridiques applicables mais aussi aller au-delà et investir davantage dans le capital humain, l'environnement et les relations avec les parties prenantes » [5] Commission Green Paper 2001. «Promoting a European... $\lceil 5\rceil$. Cette notion de capital social est également précisée: il s'agit de «l'ensemble des valeurs communes et des relations de confiance au sein d'une communauté ». Ce capital social «constitue le préalable indispensable à la coopération et à l'organisation d'activités humaines y compris dans le monde des affaires et «peut être transformé, constitué et reconstitué comme le capital financier» [6] Commission Green Paper 2001. «Promoting a European... [6]. Nous pouvons considérer, en nous appuyant sur les conclusions de Bender et Pigeyre (2003), que la diversité du genre représente un élément clef de ce capital social. Néanmoins, il apparaît important de souligner dans ce contexte, que la logique qui consiste à inscrire la diversité du genre au sein de la RSE, ne doit pas s'appuyer uniquement sur un paradigme économique. Celui-ci peut être facilement remis en cause La diversité du genre mérite au contraire de s'inscrire dans une dimension sociétale plus large, relevant d'une volonté politique (Capron M, Quairel -Lanoizelée 2004), voire d'une volonté de justice.

\section{2 - Un discours qui cache une réalité plus contrastée}

Malgré les évolutions positives et les discours largement relayés par les medias et les entreprises et en dépit d'une législation en faveur de l'embauche et de la promotion des femmes, les inégalités demeurent et l'évolution reste lente, notamment en France. Les femmes représentent 6,1\% des membres des organes de direction et de contrôle [7] Pétrovic, A.( 2006). Hommes et Expertise. La Tribune.... [7]. Sonia Bonnet-Bernard, associée chez Ricol Lasteyries et associés, souligne que quarante ans après l'instauration de la mixité au sein des grandes écoles, les résultats restent bien en deçà des attentes, hormis quelques «figures emblématiques ». Malgré un recrutement équilibré, le déséquilibre se marque après 35 ans, du fait d'un éventuel «machisme ambiant» mais aussi par une articulation des contraintes de vie 
professionnelle et de vie familiale que les femmes restent majoritaires à assumer [8] Pétrovic, A.( 2006). Hommes et Expertise. La Tribune.... [8]. Une étude présentée par l'Ecole Supérieure de Sciences Economiques et Commerciales (ESSEC) en 2006, montre que les disparités demeurent et que la diversité du genre, au moins dans l'approche de parité, peine à se mettre en place. Les différences de rémunération restent de $15 \%$ en moyenne. Chiffre surprenant si on le rapproche du nombre de femmes titulaires d'un doctorat (les femmes représentent 41\%) ou d'un mastère (les femmes représentent 58\% des mastères) [9] de Kergoren, Y. 2006. Organisation. La Tribune. 20... [9]. Sans doute, les différents dispositifs mis en place par le législateur ou certaines entreprises parmi les plus avancées, se heurtent-ils aux pesanteurs de la société française [10] Brillet, F. (2007). Management. Personnel. Février... 「10]. En effet, selon une autre étude menée par le réseau Grandes Ecoles au Féminin (GEF) en 2006, auprès de 7200 diplômées ( $35 \%$ de femmes) des grandes écoles françaises, seul 1/3 des femmes cadres ne se considèrent pas victimes de discrimination professionnelle. La question de la rétribution est la plus sensible ou symbolique, et ce dès l'embauche. On constate chez ces diplômées un sentiment d'injustice profond, d'autant que comme le précise l'étude ces «a priori » et ces discriminations ne sont pas reconnues par leurs collègues masculins [11] Perrote, D. (2007). Social. Les Echos. 8 février. [11]. L'INSEE dans sa dernière étude sur la stagnation de l'écart des salaires femmes/hommes, conclut à la responsabilité du poids des normes sociales [12] Launay, P. (2007). Dépêche l'AEF (Agence Education,... 「12]. Dans un rapport récemment paru, le Conseil Economique et Social souligne les facteurs d'inégalités persistants et propose d'instaurer, au vu de la situation, des «objectifs chiffrés de représentations équilibrées des femmes et des hommes dans les instances dirigeantes. » (Rapport CES 2007). Les quotas seraient-ils donc un mal nécessaire?

Les facteurs explicatifs sont étudiés de plus en plus finement par les chercheurs et chercheuses. Ils relèvent, comme l'indique le Conseil Economique et Social, de facteurs éducatifs et socioculturels, de pratiques organisationnelles communes à la majorité des organisations structurées en fonction de la seule approche androcentrée. Ces facteurs combinés pèsent doublement sur les conduites et comportements spécifiques des femmes elles-mêmes. (Rapport CES 2007). Il en ressort que nombre de bastions restent à conquérir et d'idées reçues à supprimer. Nous en citerons quelques uns : le mythe du salaire d'appoint, la précarité des emplois, l'orientation sexuée dès la fin des études secondaires et le cantonnement dans des professions dites «féminines » souvent peu rémunératrices, l'inégalité face au système de retraite, des rôles domestiques toujours stéréotypés, sans compter le domaine de la santé qui continue d'ignorer la prise en compte du genre en matière de santé (Gorbatko et Gelonek 2007). La politique enfin qui reste comme le souligne l'historienne Michelle Perrot, le «cristal de la virilité » dans la société française (Rapin 1999). 


\section{2 - Des réussites indéniables pourtant...}

Malgré ce constat mitigé sur l'évolution de la diversité du genre et du développement de l'égalité professionnelle femme/homme, certaines entreprises agissent. Comment font-elles ? Quels sont les facteurs qui expliquent la réussite de l'intégration de la diversité du genre ? Comment ces entreprises arrivent-elles à dépasser le discours et à inscrire dans leurs actes un nouveau paradigme culturel ? Les entreprises engagées dans la démarche du Label Egalité [13] Le Label Egalité a été mis en place fin 2004, à l'initiative... [13] mettent en place de bonnes pratiques envers les femmes que l'on peut classer en 7 catégories principales: pratiques de sensibilisation et de formation, pratiques de recrutement, pratiques de promotion, d'organisation du travail, de mobilité professionnelle des femmes, pratiques d'assistance personnelle afin de concilier vie professionnelle et vie familiale (ces mesures pouvant s'adresser aux salariés hommes également), et enfin pratiques de gestion des temps de carrière des salariés féminins notamment en cas de congés maternité ou parental (de Bry F, Ballet J.2006). Ces pratiques relèvent principalement de l'approche Egalité des chances/affirmative action et de mesures catégorielles, même si de plus en plus, l'ensemble des salariés est concerné. Néanmoins, le changement structurel éventuel de l'entreprise ne se mesure qu'à long terme, Bender et Pigeyre soulignant que l'impact en terme de gestion se mesure sur un plan qualitatif, au niveau du vécu des personnes au travail (Bender et Pigeyre 2003).

\section{1 - L'exemple de Eau de Paris}

Nous réalisons actuellement une étude qualitative auprès des salariés de l'entreprise SAGEP/ Eau de Paris. Cette étude de cas s'inscrit dans une démarche exploratoire, dans le cadre d'une thèse en gestion sur l'intégration de la diversité du genre par le management de l'organisation, mais également par les salariés. Eau de Paris qui s'est engagée dans cette démarche depuis 2001 et a fait partie des premières entreprises labellisées (15 décembre 2004) [14] Orse (2004). Répertoire des accords d'égalité prof... [14] est une entreprise basée en région parisienne qui produit et transporte l'eau de la Ville de Paris. Son statut est celui d'une Société d'Economie Mixte (SEM), concessionnaire du service public de l'eau, dont l'actionnaire principal est la Ville de Paris. L'effectif de l'entreprise est de 600 salariés dont $60 \%$ d'agents de droit privé et $40 \%$ d'agents détachés de la Ville de Paris, et $25 \%$ de salariés féminins (Source : Eau de Paris) [15] Roumier, A. (2007) Entretien du 12 février. [15]. Le choix de l'entreprise, pour cette recherche, s'est fait en fonction de plusieurs critères: un secteur d'activité technique et des métiers essentiellement masculins, une entreprise engagée dans la démarche depuis plusieurs années, et enfin une entreprise labellisée. En ce qui concerne le recueil des données, nous avons privilégié deux sources parmi celles définies par Yin (1994) [16] Yin (1994) définit six sources de données mobilisables... [16], les entretiens individuels centrés et la documentation sur l'entreprise et sur son organisation. La principale source est constituée par les premiers entretiens qui se sont déroulés à l'aide d'un guide d'entretien (questionnaire) et enregistrés. Un guide 
d'entretien spécifique a été élaboré pour chacune des populations, avec des questions en miroir qui permettront une analyse des convergences et des divergences entre les objectifs du management et la perception des salariés. Les données recueillies ont été retranscrites et sont en cours d'analyse. L'échantillon actuel est constitué par deux populations : « salariés » et « dirigeants ». Onze entretiens ont déjà été réalisés en février 2007. Les prochains entretiens sont planifiés en avril 2007. Nous présentons très brièvement les caractéristiques de ce premier groupe.

\begin{tabular}{|c|c|c|c|}
\hline \multirow{8}{*}{ Répartition femmes/hommes } & Femmes & 3 & $38 \%$ \\
\hline & Hommes & 8 & $62 \%$ \\
\hline & Qualification & & ore d'interviews \\
\hline & D2 & 3 & \\
\hline & D1 & 2 & \\
\hline & C2 & 1 & \\
\hline & C1 & 2 & \\
\hline & B2 & 3 & \\
\hline
\end{tabular}

Les résultats présentés sont sommaires, mais ils permettent d'ores et déjà, de mettre en lumière des éléments clés du «modèle émergent » tels que les définissent Ely et Thomas (1996), appliqués à la diversité du genre :

- l'implication et l'engagement sincère de la direction,

- une culture d'entreprise favorable qui favorise des attentes de compétences au niveau de chacun,

- une orientation Qualité dans tous les secteurs de l'entreprise,

- une culture qui favorise l'ouverture, permettant le débat et la discussion, une culture qui permet à l'individu au sein de l'entreprise de se sentir « respecté »,

- une perception claire et largement partagée par l'ensemble des salariés des buts et des missions de l'entreprise,

- une structure non bureaucratique, de type égalitaire.

\section{2 - De nouveaux secteurs cherchent à intégrer la diversité du genre}

De nouvelles entreprises s'engagent vers la recherche de la diversité du genre et l'intégration des femmes à tous les niveaux de l'entreprise, dans une logique de féminisation des métiers.

L'industrie a entamé son évolution vers la féminisation des effectifs dès 2002. Le cas du secteur du BTP est à cet égard révélateur. Le BTP, bastion masculin par excellence dans notre 
société, montre que les lignes de clivage traditionnel métiers masculins/métiers féminins évoluent, même lentement, car la ségrégation des métiers masculins et féminins ne disparaît pas si facilement.

\subsection{1 - Approche historique du travail des femmes et métiers masculins et féminins}

«Ce ne sont pas seulement les métiers qui ont un sexe. Le sexe de celui qui occupe tel ou tel type de poste constitue un marqueur durable de la représentation de l'emploi » Cornut-Janin (2000). Les femmes ont toujours travaillé et les travaux des historiennes et des historiens le démontrent. Les périodes historiques antérieures à l'industrialisation massive ou le développement de l'emploi salarié dans les services montrent qu'à la campagne comme à la ville, les femmes exerçaient de nombreux métiers. Le Moyen Age notamment permet aux femmes, sous le contrôle des corporations, d'intégrer les métiers de l'orfèvrerie ou du travail de la soie. C'est le XIXème siècle, qui va établir la notion de métier féminin, lors des premières réflexions sur le marché du travail et lier le concept avec les supposées qualités féminines (Duby, Perrot, Farge 1993). «Les métiers propres aux femmes » tels que les définit Paul Leroy Beaulieu [17] Paul Leroy-Beaulieu (1843 -1916), économiste franç... [17] se retrouvent dans les secteurs de l'enseignement du fait de son lien avec les enfants et du tertiaire (banque, administrations publiques des postes et des télégraphes). Le lien entre métiers féminins et qualités féminines existe toujours dans les mentalités, même au XXIème siècle. Le métier féminin en tant que tel, n'existe pourtant plus dans la législation depuis 1945. Cette perception de métiers «féminins » et donc de métiers «masculins » évolue avec l'histoire et s'articule avec la construction sociale du rapport des sexes. Elle trouve sa source dans « le symbolique, le mental, le langage » [18] Maurice Godelier cité par Marie Durut Bellat. 1995....「18] . Les frontières établies entre ces métiers par les employeurs (et également plus généralement la société dans son ensemble) sont celles de la masculinité et de la féminité. Aux femmes, les secteurs du social, de la santé, de l'enseignement et de la bureautique, sans oublier quelques métiers créatifs comme le design et la décoration. Aux hommes, les secteurs à forte pénibilité, dangereux ou traditionnellement masculins et notamment le bâtiment et les travaux publics. On associe souvent la technicité et l'outil à la masculinité. Certains métiers échappent à cette catégorisation. Ce sont le plus souvent des métiers «modernes » (informatique, marketing) qui n'apparaissent pas, du fait de leur nouveauté, comme « sexo-spécifique ».

Les statistiques montrent la forte concentration horizontale des métiers des femmes dans le tertiaire et le secteur des services (Fortino 2002). Ce développement du tertiaire a permis la croissance de l'emploi féminin et l'évolution entamée durant la période de l'entre-deuxguerres se poursuit aujourd'hui. Le secteur tertiaire emploie $85 \%$ de la population active féminine pour seulement $60 \%$ de l'emploi masculin. $3 / 4$ des salariés sont des femmes dans les secteurs de l'éducation, de la santé et de l'action sociale. A l'inverse, la Construction n'occupe que 9,5\% de femmes, soit un poste sur 10 [19] INSEE (2003). Enquête Emploi. [19]. Les statistiques de l'INSEE prouvent que hommes et femmes n'occupent pas les mêmes métiers : 
on retrouve les hommes dans les catégories techniciens ou agents de maîtrise, les femmes restant cantonnées dans les professions intermédiaires de la santé et du travail social (INSEE 2004). Ce sont ces clivages qui commencent à se fissurer. En témoignent les chiffres avancés par l'Union des Industries des Métiers de la Métallurgie (UIMM), les femmes représentent $20 \%$ des salariés dans le secteur des métaux, $14 \%$ dans le secteur automobile, et $6 \%$ seulement dans le ferroviaire et l'aéronautique. Elles se concentrent dans l'encadrement mais restent peu présentes chez les ouvriers et les employés techniciens (Montaigne 2007). Développer l'emploi féminin ouvrier dans la métallurgie notamment reste très difficile. L'entreprise Turbomeca, spécialisée dans la fabrication de turbines pour hélicoptères, avions et missiles n'a vu progresser son effectif féminin que de $1 \%$ entre 2000 et 2006 passant de 12 à $13 \%$. Les femmes constituent $1 \%$ des effectifs ouvriers. "Les conditions de travail en atelier ne justifient plus par leur pénibilité qu'il n'y ait pas de femmes, mais on ne trouve pas de candidates » souligne Philippe Boulan, DRH de l'entreprise [20] Reiss.C (2007). AEF. Dépèche 73483. [20]. Dans le BTP, la proportion des femmes, parmi les ouvriers est de 1,3\% [21] Le BTP au féminin (2007). www. batiactu. com $[21]$. Comme l'indique Hervé Maligre, responsable d'un centre de formation sur les métiers de la mécanique : «ce n'est pas parce qu'un métier est technique ou un peu rude qu'il ne convient pas aux femmes » [22] Cité par Montaigne. C. (2007). Ces derniers bastions... [22]. Le frein essentiel reste les mentalités mais également dans le fait que les filles ne se dirigent pas spontanément vers ces métiers, compte tenu des pratiques d'orientation très fortement sexuées qui existent actuellement. Les préjugés demeurent tenaces même si selon l'UIMM, seuls $5 \%$ des métiers ne seraient pas accessibles aux femmes [23] Montaigne. C. (2007). Ces derniers bastions masculins... [23]. Le progrès technologique, l'évolution des conditions de travail et les études de postes permettent de dépasser la question de la force physique qui devient un critère moins discriminant. Le BTP constitue un des exemples les plus intéressants d'évolution et d'efforts vers ce nouveau paradigme de la diversité.

\subsection{2 - La révolution du secteur du BTP : la féminisation des métiers}

Le BTP devra faire face dans les prochaines années à des départs massifs en retraite qui ont déjà commencé. L'enjeu pour ce secteur consiste à présenter des perspectives attrayantes d'évolution de carrière. Les entreprises de BTP sont invitées à montrer une nouvelle image d'elles-mêmes, tant en matière de savoir-faire que d'évolution managériale, auprès de deux populations cibles : les jeunes et les femmes. En ce qui concerne ces dernières, le secteur s'engage donc dans une approche radicalement nouvelle d'intégration des femmes à des postes traditionnellement masculins. Cette approche est facilitée, on l'a vu, par la modification de la pénibilité des emplois, l'amélioration des conditions de travail et l'évolution des postes. On peut citer également un meilleur accès des femmes aux formations techniques (Derrez 2007). La Fédération Française du Bâtiment (FFB) souhaite multiplier par trois le nombre de femmes sur les chantiers et en ateliers pour atteindre en 2009, 30000 salariées, dont 20000 sur les chantiers [24] Regy.S.( 2007). www. lemoniteur-expert. com [24]. 
L'objectif de la FFB ne pourra être atteint néanmoins que si les représentations sociales du secteur, de l'école à l'entreprise, arrivent à se modifier en profondeur. Il semble, si l'on analyse les propos de Laurent Debord, secrétaire général de la FFB Gironde [25] Le BTP au féminin (2007). www. batiactu. com $[25]$, que les qualités (féminines ?) «que les hommes ne possèdent pas, comme le souci de la finition, du détail» feront merveille sur les chantiers. Reste à convaincre les intéressées.

Nous pouvons nous appuyer sur une étude «Femmes dans le Bâtiment » réalisée par la société EMSIS qui synthétise divers travaux de 2003 et 2004 sur l'image et les représentations sociales de la Construction. Ces enquêtes ont été réalisées auprès de femmes, du grand public, de responsables d'entreprises et de salariés du secteur. Cette étude montre que le secteur du bâtiment est perçu comme particulièrement hétérogène rassemblant aux côtés de quelques grands groupes internationaux de Construction, une myriade de petites structures de type artisanal. A cette hétérogénéité des acteurs, on peut rajouter la perception floue de métiers extrêmement divers, à l'identité forte et aux savoir-faire clairement identifiés et nettement associée à la masculinité. Ce secteur, en ce qui concerne les adolescentes et les femmes, n'appartient pas au champ des métiers spontanément envisagés pour les filles. L'étude et les recherches effectuées auprès du système éducatif le confirment : le manque de connaissance des jeunes filles sur le secteur et les métiers offerts, ainsi que des perceptions particulièrement négatives, les éloignent du secteur du BTP. Ces métiers sont vus comme trop manuels, trop physiques, trop salissants, mal payés et surtout réservés aux garçons. Chez les femmes plus âgées, la représentation change légèrement et évolue plus positivement en évoquant la noblesse et l'utilité de la tâche, les possibilités de mobilité, de travail en équipe et d'autonomie. Les métiers restent néanmoins qualifiés «d'ingrats » à cause des intempéries. Il faut noter également que le déficit d'information et d'image de ce secteur se retrouve auprès des professionnels des services éducatifs et de l'emploi. Par contre, parmi les salariés du secteur (hommes ou femmes), on peut faire ressortir l'existence d'une « culture métier forte et valorisante », le sentiment d'appartenir à une profession génératrice de satisfactions profondes. Les bouleversements qui s'opèrent actuellement dans les organisations en général, se retrouvent dans les entreprises du secteur. Le poids nouveau des approches relationnelles et organisationnelles, les modifications des techniques, le développement croissant des technologies de l'information et de la communication modifient radicalement l'image classique du métier stéréotypé du BTP. «L'homme de marbre » [26] L'homme de Marbre. 1976. Film de Andrzej Wajda. [26] a perdu son sens emblématique. Nous pouvons néanmoins nous interroger sur les motivations et les attentes suscitées par cette demande de «féminisation » dans ces métiers, les avantages «perçus ou imaginés» [27] Emsis (2004). L'image et les représentations sociales... [27] qu'apportera l'intégration des femmes. L'étude souligne que le contexte reste celui d'une intégration « limitée » qui n'envisage pas une présence massive. La perception des femmes qui exercent ces métiers, fait référence à la force de caractère et de personnalité qu'exige pour une fille le fait de refuser les schémas traditionnels, ou pour une femme adulte de modifier une trajectoire professionnelle. La compétence égale ou supérieure 
à celles des hommes est également citée. On retrouve de manière symptomatique la référence aux supposées qualités féminines (qualités de méthode et de soin, esthétisme) associée à une intelligence d'organisation des modalités du travail. Sans compter une saine émulation qui favoriserait la qualité globale du travail et la motivation des salariés masculins. L'intégration des femmes au sein de ces métiers doit permettre également une amélioration des comportements et des conditions de travail, profitant aux salariés dans leur ensemble. L'effet positif de surprise et d'image est également souligné. Ces thématiques sont en cohérence avec les problématiques de l'approche «business case» que développent actuellement les entreprises du BTP: que peut gagner l'organisation à intégrer et à valoriser une main d'œuvre féminine? Les approches empiriques, réalisées dans d'autres secteurs «techniques » et «masculins» engagés depuis quelques années dans des processus de féminisation des effectifs, confirment ces attentes. Nous ferons référence à l'étude qualitative en cours au sein de l'entreprise Eau de Paris que nous avons évoquée précédemment. Les premières interviews réalisées permettent de retrouver ces thèmes cités par les salariés masculins et féminins de l'entreprise interrogés sur leur vécu, notamment en termes d'amélioration des comportements au travail et des conditions de travail, profitant aux salariés dans leur ensemble.

Les freins restent cependant nombreux. L'enseignement professionnel n'a pas en France une image suffisamment valorisante et les modèles parentaux, en ce qui concerne les filles, restent classiques et centrés sur les métiers «féminins ». Le manque d'information et la représentation négative des métiers alimentent les préjugés et figent les images des rôles sociaux dans une dualité féminin-masculin. Les chefs d'entreprises eux-mêmes restent réservés sur l'intégration des femmes malgré le besoin de main-d'œuvre. La logique invoquée est celle d'une adaptation obligatoire des femmes à l'organisation et non le contraire. Les contraintes pratiques (vestiaires et toilettes séparés) sont considérées comme un poste de coût supplémentaire. On retrouve principalement dans les freins exprimés, l'inquiétude que les hommes puissent se sentir «discriminés » par le traitement favorable fait aux femmes, le doute toujours sur la capacité de ces dernières à fournir un travail équivalent à celui d'un homme et les réticences classiques désormais, liées à la maternité, à la conciliation vie professionnelle et vie familiale, toujours perçue comme une problématique féminine par les hommes et les femmes d'ailleurs. Ces préjugés toutefois sont d'autant plus fortement exprimés que l'âge du responsable d'entreprise est élevé. Ce qui laisse augurer d'une évolution favorable auprès des générations plus jeunes. Enfin l'opposition des conjointes dans les entreprises artisanales n'est pas à négliger ainsi que celle des femmes déjà en place. Les résultats provisoires d'une étude [28] Etude menée en 2005 par le Centre régional d'orientation... [28] réalisée auprès de 99 femmes en France par l'AFPA montrent que la parité peut avoir un effet négatif sur la volonté des femmes de rester dans une profession atypique ou masculine et diminuer ce «sentiment d'exception » d'être la seule à exercer ce métier dans l'entreprise [29] Baron F. (2006). Métiers atypiques. AEF. Dépêche 6...「29]. Néanmoins, les expériences se multiplient et chaque nouvelle expérience participe d'une contagion vertueuse de changement des mentalités. Le BTP a obtenu en octobre 2006 son premier Label Egalité [30] Ce label est obtenu 
après dépôt d'un dossier de candidature... [30]. Il s'agit de l'entreprise Laine-Delau, PME parisienne de 260 salariés, réalisant un chiffre d'affaires de plus de 50 millions d'euros et spécialisée dans la restructuration lourde, la construction neuve et la rénovation du patrimoine: aujourd'hui il y a une femme ouvrière dans leur effectif... D'autres entreprises ont également décidé de diversifier leur recrutement et de «sourcer» des femmes. C'est le cas de l'entreprise CARI, située à Nice, qui développe une approche en amont, de formation et de recrutement, notamment en partenariat avec la société de recrutement Adia.

\section{3 - La réalité du terrain : l'expérience de CARI}

\section{1 - Présentation de CARI}

CARI est une entreprise de Construction et de Services. Sa principale activité, le gros œuvre en Bâtiment et Travaux publics, se déploie sur l'ensemble du territoire français, au travers de 8 Directions régionales. Avec ses 2350 collaborateurs et collaboratrices, un chiffre d'affaires de 418 millions d'euros a été atteint en 2006 : depuis deux ans, l'entreprise poursuit une croissance de plus de $20 \%$. Cette évolution se répercute également au niveau des effectifs : 900 collaborateurs et collaboratrices ont été recrutés l'année dernière, grâce au cumul de croissance interne et externe. Si le secteur du Bâtiment et des Travaux Publics (BTP) se porte aujourd'hui très bien, CARI présente une croissance supérieure à la moyenne du marché et occupe un positionnement singulier dans son métier. Le secteur du BTP est essentiellement représenté par trois majors (qui sont également leaders au niveau mondial) et une myriade de petites entreprises. Les entreprises de la taille de CARI, de taille intermédiaire, sont en effet très peu nombreuses sur le marché français. Enfin, la Responsabilité sociétale constitue un des axes principaux de la stratégie de l'entreprise : son engagement aussi bien au niveau de l'environnement, de la sphère économique et du social est aujourd'hui une priorité considérée comme un gage d'avenir pour la structure de l'organisation ainsi que pour le secteur de la Construction en général.

\section{2 - L'expérience menée chez CARI : une logique de RSE}

Il est important de rappeler ici le contexte de l'emploi qui perdure dans le secteur depuis quelques années: alors que le marché de la Construction se porte bien, il souffre paradoxalement d'une forte pénurie de personnel qualifié, aussi bien au niveau des postes d'ouvriers que des postes d'ingénieurs. D'une part, le secteur a une mauvaise image qui limite considérablement son attractivité, comparé à d'autres secteurs, notamment à des segments plus récents tels que l'informatique ou les nouveaux médias qui attirent aujourd'hui toutes les attentions grâce à leur image innovante dans les représentations collectives. D'autre part, les profils des personnes arrivant aujourd'hui sur le marché de l'emploi se sont profondément modifiés en matière d'aspiration. De ce fait, les offres d'emploi sont aujourd'hui très nombreuses chez CARI, mais également sur l'ensemble du marché de la construction. Et les 
difficultés pour recruter nécessitent d'imaginer des solutions nouvelles dans un contexte fortement concurrentiel. Il convient ici de relever que les niveaux de rémunération ont connu également une croissance très forte, qui se poursuit aujourd'hui. Ce qui implique de réfléchir à toutes les plus-values qu'une entreprise peut apporter au-delà de la rémunération et des avantages matériels. Dans ce contexte, en 2004, un travail a été mené chez CARI en vue d'anticiper les besoins de recrutement liés à la croissance de l'activité. Le choix a été fait d'orienter spécifiquement le recrutement sur trois publics et, donc de réfléchir à une stratégie avec des outils adaptés au contexte et à l'entreprise. Les trois publics visés sont : les jeunes, les seniors et les femmes.

Le travail avec les jeunes vise tout d'abord à développer l'attractivité des métiers de la Construction. Il s'agit ensuite d'adapter les parcours d'intégration, aussi bien en matière d'insertion pour des personnes éloignées de l'emploi ou à faible niveau de qualification (voire sans qualification) que pour les personnes diplômées, en les fidélisant par la mise en place de parcours initiatiques individualisés. La question du public senior renvoie au contexte du papyboom et à la nécessité de transmettre le savoir-faire, le savoir être et la culture métier d'une génération fortement représentée qui est en train de partir à la retraite, envers les jeunes qui entrent dans le secteur et dans l'entreprise. Ce travail avec les jeunes et les seniors participe d'une problématique commune à ces deux publics. Par ailleurs, le secteur a toujours connu une diversité des âges forte, avec un système traditionnel de transmission des savoirs : il s'agissait ici de réactiver des méthodes propres à la culture du métier et de l'entreprise et de les intégrer dans des processus obligatoires et rationalisés. Il convient de relever que la pyramide des âges dans l'entreprise présentait deux ans auparavant un pic très élevé au niveau de la génération des plus de 50 ans. A l'issue de deux années d'un travail considérable en matière de recrutement, la tendance a pu être renversée.

Concernant le public féminin, la stratégie est à la fois similaire et différente. Similaire puisqu'il s'agissait d'attirer vers l'entreprise CARI un public qui n'était pas intéressé par les métiers offerts, au même titre que les jeunes et le public en général. Différente du fait de l'absence quasi totale de représentativité de ce public, au sein du milieu ouvrier du gros œuvre en France. Une analyse comparative a permis d'identifier de nombreux pays en Europe où les femmes étaient représentées au sein de ce métier. L'objectif était ici de signifier l'ouverture de CARI à un public féminin et de proposer des parcours de recrutement, d'intégration et de formation permettant d'imaginer que ce public rencontrerait des opportunités similaires à celles des hommes dans les métiers de la Construction. Il s'agissait ensuite de communiquer directement auprès du public concerné. Il est important de rappeler qu'au moment où ces choix ont été opérés, une campagne médiatique forte était orchestrée, notamment dans le cadre du référendum européen, au sujet de l'arrivée d'une main d'œuvre communautaire annoncée comme qualifiée et bon marché, assortie de sollicitations nombreuses de la part d'entreprises qui se sont positionnées sur ce créneau de manière exclusive, notamment par rapport aux pays de l'Est nouvellement entrés dans l'Europe. Si le secteur de la Construction 
a contribué à des vagues massives d'immigration, les conditions d'obtention d'un permis de travail ne permettent plus depuis des années d'avoir recours à ce mode de recrutement. L'espace communautaire, et l'espace Schengen en particulier, offrent différentes solutions en matière de mobilité, mais qui n'ont rien à voir avec ce qui a pu être mis en œuvre il y a quelques décennies. Enfin, il convient de mentionner également que plus de la moitié des ouvriers chez CARI sont de nationalité étrangère et que la majorité a été recrutée directement sur le territoire national [31] CARI est fusion d'entreprises régionales dont les spécificités... [31]. L'entreprise a en effet choisi aujourd'hui, dans un environnement de l'emploi dominé par un fort taux de chômage, de travailler au plus près des territoires, là où elle est implantée, et de s'axer à ce moment précis sur ces trois publics prioritaires [32] Durant cette même période où le projet de féminisation... [32]. Enfin, il a été créé il y a deux ans au sein de la Direction des Ressources Humaines, un pôle Responsabilité et Innovation Sociales, qui coordonne l'ensemble des actions mises en œuvre au niveau national. Les principaux projets menés par l'équipe sont: l'insertion, secteur dans lequel l'entreprise s'est investie de tout temps et qui nécessite aujourd'hui une adaptation certaine compte tenu des aspirations nouvelles des publics; l'intégration des travailleurs handicapés, qui est aussi un domaine historique et enfin le projet de féminisation des effectifs ouvriers, le plus récent de tous.

\section{3 - La démarche Egalité Professionnelle chez CARI}

Le développement de ce projet d'intégration de femmes au sein de la population ouvrière a été mise en œuvre au sein un dispositif plus général touchant à l'égalité professionnelle femmehomme au sein de l'entreprise. En parallèle des actions opérationnelles menées, une étude de la place des femmes au sein de l'entreprise CARI a été réalisée : le support initial en a été la production du Rapport de situations comparées qui a permis de définir des indicateurs concrets et statistiques, nourrissant la réflexion dans la mise en ouvre de ce projet [33] Il est important de mentionner que les représentants... [33].

Si le secteur affiche 9,5\% d'effectif féminin, ce public est aujourd'hui quasiment absent de la population ouvrière. La Fédération Française du Bâtiment qui mène une campagne importante sur la féminisation des métiers témoigne d'expériences réussies en matière d'intégration de femmes dans les métiers du second œuvre mais CARI est la seule entreprise, à ce jour, à être parvenue à développer un projet spécifique avec des résultats de cette ampleur en matière de gros œuvre. Les femmes présentes dans le secteur occupent des postes au sein des services transverses, souvent même des postes de direction (ressources humaines, communication...) mais ne sont pas représentées dans les directions opérationnelles même si au cours de ces dernières années, elles se retrouvent plus fréquemment dans l'encadrement chantier et les services études. Il n'y a cependant pas encore de parité à ce niveau. Chez CARI, les femmes constituaient il y a deux ans, $10 \%$ de l'effectif global, et leur répartition par poste correspondait à la situation générale du secteur du BTP. Comprenons ici qu'aucune femme n'était représentée dans la population ouvrière. Fin 2005, CARI comptait 2 ouvrières et début 
2007, elles étaient 37 à occuper des postes de coffreuse, maçonne, grutière, conductrice d'engin, manœuvre, ... fruit d'un travail ambitieux qui se poursuit aujourd'hui et vise à ce que 100 ouvrières travaillent sur les chantiers de CARI d'ici la fin de l'année 2007. Une femme a également été nommée il y a deux ans à la Direction Générale (composée de 6 personnes au total). Cette percée du «plafond de verre » représente un acte fort en matière d'accès des femmes au top management de l'entreprise. Cette nomination est importante car elle permet de créer des modèles pour les femmes au sein de CARI mais également d'affirmer le désir et la possibilité de mixité à toutes les fonctions.

\section{4 - Les difficultés rencontrées}

Elles se concentrent autour de deux problématiques, celles liées aux phénomènes des résistances masculines et celles liées aux difficultés de trouver la ressource recherchée.

\subsection{1 - Les résistances masculines : la diversité sexuée est la plus difficile à être acceptée dans ces métiers}

La spécificité du secteur du BTP est de présenter des effectifs avec une très forte diversité : qu'il s'agisse d'âge, de nationalités, de cultures, de religions... les équipes œuvrant sur les chantiers reflètent une vision cosmopolite de l'homme. Et c'est bien là également sa limite car il ne s'agit que d'hommes. En effet, les effectifs sont exclusivement masculins et la présence de femmes était exceptionnelle il y a encore quelques années. Cette situation qui se modifie chez CARI notamment, devrait entraîner des répercussions sur l'ensemble du secteur. Les femmes étaient absentes de l'encadrement chantier mais le processus de féminisation des postes avait déjà été amorcé. La décision de la Direction Générale de CARI d'intégrer des femmes sur les chantiers a fait l'objet d'une campagne de communication très importante au sein de l'entreprise (annonce dans le Journal interne, lors des séminaires...). Celle-ci a été reçue avec une grande surprise, énormément de scepticisme et surtout de nombreuses réactions de rejet. La première réaction fut l'incompréhension, génératrice d'une forte hostilité vis-à-vis de ce projet. Avec le recul de ces deux premières années, force est de constater que ce projet a fait l'objet d'une communication forte, mais non assortie d'effet immédiat au sein des équipes. Ce n'est, en effet, qu'un an plus tard, que les femmes sont arrivées sur les chantiers dans la plupart des sites, Carros ayant été le site pilote. Ce discours, non accompagné par des actes suscita une controverse au sein de l'entreprise CARI, visant à remettre en cause la pertinence et le sens même du projet. Cela n'a empêché ni sa mise en place, ni sa réussite mais a nécessité un travail considérable d'échange avec les équipes de chantier, afin de trouver des relais et des appuis dans les équipes et mettre en place de véritables processus pour optimiser l'intégration et la pérennisation de ces postes. On peut considérer que l'arrivée des femmes a été perçue comme celle de l'ultime public non représenté, constituant à la fois un défi pour les groupes d'hommes, mais également une limite susceptible de remettre en cause la cohésion d'un ensemble. A ce stade du projet, c'est 
surtout la notion de groupe sexué qui peut être pointée : certains évoquent la «Maison des Hommes » et la «Maison des Femmes » pour identifier les différents groupes de rattachement sexués au cours de l'éducation et du passage de l'enfance à l'âge adulte (Godelier 2003, Weltzer-Lang 2002). Mais dans le monde du travail persiste également ces communautés sexuées qui disposent de leur propre mode de fonctionnement, de reconnaissance et d'échange. Et lorsque l'on évoque la Construction en France, on se situe au cœur des métiers d'hommes... L'arrivée de femmes dans la «Maison des Hommes » remet en cause leur système de cohésion et bouscule leur propre culture. Cette irruption des femmes est perçue immédiatement comme une agression visant à remettre en cause le monopole des hommes et du système : chose difficilement envisageable dans leur imaginaire et dans leur présent.

Des systèmes de résistances se sont donc mis en place, témoignant des résistances masculines plus générales au changement. Tout d'abord un système assez classique de dénigrement s'appuyant sur bon nombre de moyens propres à la domination masculine (Bourdieu 2002). Une des caractéristiques principales en a été la notion de collectif et de public : en effet, on a pu distinguer très spécifiquement la logique du discours officiel à tenir, surtout devant les autres. Ensuite, on a constaté que dans des relations individuelles, les avis devenaient beaucoup plus nuancés. Les principaux préjugés identifiés portaient sur : la limite en matière de force physique («elles ne peuvent pas y arriver, elles n'en sont pas capables »), l'absence de légitimité au milieu des hommes et sur un chantier («elles n’ont pas leur place sur les chantiers, c'est un monde d'hommes »), les bouleversements supposés dans les équipes et les risques de perturbation (portant aussi bien sur la nécessité de les « aider » car elles «n'allaient pas y arriver» que sur les absences liées à la maternité. Parmi les autres «phantasmes négatifs » qui ont pu être recensés, on peut citer également le risque de désorganisation et de baisse de productivité, les perturbations qu'une femme peut générer dans un groupe, perçue ici comme un élément qui bouleverse l'ambiance, les surcoûts d'un tel projet («il va falloir leur installer des vestiaires et des sanitaires, ça va nous coûter cher » [34] Il s'agit là d'une obligation légale dont le coût est,... [341) jusqu'aux poncifs sur la place des femmes dans les sphères professionnelles et privées («elles feraient mieux de rester à la maison»). Le corpus même de cet argumentaire démontre la crainte du changement mais également une appréhension forte en matière de concurrence, dans la remise en cause du monopole masculin.

\subsection{2 - La difficulté de «sourcer les femmes» sur des métiers considérés comme masculins et non valorisés}

Les difficultés rencontrées au niveau du «sourcing » relèvent d'un aspect général et d'un aspect plus spécifique à ce public. Les métiers du BTP, nous l'avons évoqué précédemment, ont aujourd'hui une attractivité très faible liée à une image négative de la Construction dans les représentations collectives (pénibilité, dangerosité, illégalité, ...). Ces différents stéréotypes réfèrent à des épisodes de l'histoire de ce secteur qui tous n'ont pas disparu. 
On relève que la grande solidarité qui règne au sein des équipes, tout comme l'aspect collectif et concret du travail, ainsi que le savoir-faire ne sont pas ou peu présents aujourd'hui dans l'imaginaire collectif. Cela rend donc difficile le «sourcing » de candidats en terme général. Les personnes qui viennent travailler dans ces métiers semblent souvent y arriver, au niveau ouvrier, comme une des dernières possibilités d'intégration professionnelle. La Construction ne constitue pas un métier vers lequel on se projette mais dans lequel les candidats semblent atterrir en dernier recours. Une des singularités du projet de féminisation est d'être très similaire au dispositif mis en place dans le cadre des différents projets d'insertion, tant du point de vue de certains profils recrutés, que dans les parcours de recrutement qu'il a fallu mettre en place. En matière de suivi également, les outils sont aussi, le plus souvent, similaires. La spécificité du projet se retrouve dans le travail de défrichage qu'il a fallu mener auprès des différents acteurs de l'emploi, aussi bien publics que privés. En effet, les équipes de ressources humaines ont entrepris une tâche qui pourrait s'assimiler à du militantisme tant le projet a surpris les partenaires institutionnels. Les résistances ont, de ce côté également, été fortes. Il a fallu convaincre les différentes structures de participer au montage d'une telle opération. La Construction offre de considérables opportunités en matière d'emploi mais la difficulté à trouver un public motivé freine nombre d'acteurs du domaine. Le projet évoqué d'intégration de femmes sur les chantiers a été parfois perçu comme un projet difficile voire irréaliste.

Une fois l'identification des bons interlocuteurs réalisée, les projets se sont mis en place. Il n'y a pas eu de partenaire unique appartenant au secteur public de l'emploi, mais une multiplicité de partenaires publics qui se sont engagés de manière participative et volontaire. Par contre, un partenariat spécifique a été signé avec l'entreprise de recrutement ADIA pour développer ce projet sur l'ensemble des initiatives projetées. Leur expertise en matière de projets intégrant la lutte contre les discriminations a été bénéfique dans la conduite des projets de CARI. Enfin, plus en amont, il convient de resituer la question du recrutement dans un contexte culturel plus général. Le domaine de la Construction correspondant dans les représentations à des métiers d'homme, les filières de formation, elles-mêmes, ne proposent pas, ou très peu, de candidatures féminines. Concernant les formations techniques, les femmes restent faiblement intégrées et pour ce qui est des filières ingénieurs, elles sont encore bien loin d'y être présentes de manière égalitaire. Plus en amont se pose la question de l'orientation scolaire et des filières professionnelles. Ces thèmes ne peuvent être dissociés du rôle joué par le corps professoral dans les conseils d'orientation, mais aussi, et c'est essentiel, par les familles et la société en général sur les représentations professionnelles genrées. Mais il s'agit-là d'un chantier que CARI n'a pas attaqué [35] Il convient de nuancer ce propos : en effet, dans le... $[35]$... La question des filières a d'ailleurs été à l'origine de la mise en place de parcours de formation interne, indispensable pour que chaque candidate puisse être qualifiée, donc reconnue, par les équipes et tout simplement au sein de la profession.

\section{5 - Comment surmonter ces difficultés ? l'approche de CARI}


La réussite du projet repose d'une part sur un management fort et valorisant et d'autre part sur le développement d'outils spécifiques mais non différenciés. La question du management apparaît centrale. Ce type de projet, et plus encore dans ce secteur de la construction, nécessite une impulsion et un soutien fort et permanent de la Direction générale. La Direction Générale ici a initié le projet, maintenu le cap et démontré sa volonté de poursuivre dans cet axe défini comme un élément prioritaire de la stratégie de l'entreprise. En effet, des objectifs d'effectifs féminins à atteindre ont été fixés annuellement et des états des lieux mensuels doivent témoigner des actions mises en œuvre dans ce sens.

La Direction générale joue donc un rôle essentiel mais la réussite du projet passe par une appropriation du projet et de sa défense par tous les échelons de la hiérarchie, et notamment les chefs d'équipes. C'est à ce niveau sans doute, que le travail de sensibilisation demande le plus d'énergie. Les directions régionales et les directeurs d'agence ne doivent pas douter du bien-fondé d'un projet dont la légitimité est largement remise en cause par les ouvriers. Pourtant, c'est à ce niveau que les freins les plus importants se sont manifestés et ont nécessité des recadrages, publics ou individuels, afin de s'assurer du soutien inconditionnel de la part de l'encadrement. La question du management reste cruciale dans le choix des équipes où des femmes vont être intégrées. Parmi les chefs de chantier, les conducteurs de travaux et maîtres compagnons, deux profils ont été sélectionnés : d'une part des managers qui ont une emprise forte sur leur équipe, qui sont respectés, et dont l'autorité n'est pas remise en cause, d'autre part des profils de managers dont la capacité à transmettre le savoir-faire, à accueillir des jeunes ou des stagiaires et à les former est connue et légitimée. Enfin, la question du management se pose directement au niveau des équipes des Ressources Humaines qui ont mis en œuvre le projet : leur intervention a nécessité une formation et un accompagnement terrain déterminants pour que ces équipes puissent être des acteurs moteurs du projet. A aucun niveau, l'appropriation du projet ne devait être remise en cause, ce qui a exigé une fermeté certaine et un engagement fort de la part de l'encadrement.

Des outils spécifiques d'intégration et de suivi ont été développés. Ceux-ci ont vu le jour dans le cadre du partenariat avec l'entreprise de recrutement ADIA avec qui l'ensemble du process (sourcing, recrutement, intégration, formation et suivi) a été défini dès le départ. Deux d'entre eux sont singuliers et viennent s'ajouter à de nombreux outils d'intégration déjà présents chez CARI et qui participaient également à la mise en œuvre du projet (le tutorat, les journées d'intégration, ...) :

- Le premier outil, en amont, est la réalisation d'un diagnostic auprès des équipes allant accueillir des femmes, afin d'identifier les leviers sur lesquels s'appuyer pour faciliter le succès du projet, désamorcer les freins existants avant l'arrivée des candidates. Ce diagnostic a été réalisé par les Agences Régionales d'Amélioration des Conditions de Travail, un organisme dont la neutralité extérieure est gage d'objectivité dans la 
restitution des résultats. Systématiquement, les conclusions ont été présentées de manière collective aux personnes qui avaient été interviewées de manière individuelle. Les groupes interrogés constituaient un panel représentatif de salariés et de représentants: ouvriers, encadrement chantier, chef d'agence, médecin du travail, représentant du personnel... Les données recueillies s'articulent autour de deux axes : des arguments plutôt défavorables à l'accueil des femmes que d'ailleurs le simple fait de les évoquer désamorce et permet de minorer leur importance mais également une analyse structurelle de l'agence et des équipes qui permet d'évaluer le management de chaque agence et d'envisager des adaptations protéiformes en fonction des conclusions.

- Pendant la phase projet, un suivi renforcé des candidates a été mis en place: tout d'abord, il a été demandé de la part de l'encadrement de relater auprès du service des Ressources Humaines, tout événement spécifique en relation avec l'intégration de chacune des stagiaires. Des points hebdomadaires ont été réalisés, à la fois par un représentant du Service Ressources Humaines de CARI et par une personne de la société ADIA [36] Le projet s'organisait autour d'une période d'environ... [36]. Cela a permis de recadrer le projet au fur et à mesure [37] Un des problèmes rencontrés et récurrents a porté sur... [37], et d'identifier les points spécifiques à traiter qu'il s'agisse de problèmes concernant les candidates, de problèmes de motivation, de problèmes techniques ou comportementaux...

Enfin, comme il a été mentionné dans l'introduction, il était important de réfléchir à des modes d'intégration adaptés mais qui ne soient pas non plus différenciés, afin de ne pas stigmatiser ces arrivées. Le processus mis en œuvre en témoigne à la fois par un travail important de la part de ceux qui l'ont coordonné, mais présenté de manière plus légère pour les équipes afin de ne pas les déstabiliser avec un projet trop lourd.

\section{6 - Les apports de l'intégration des femmes : que peut-on en dire à l'issue de cette première phase du projet ?}

Il semble qu'un des éléments qui se dégage permette de concevoir la féminisation comme un outil de performance, mais également une dimension du changement de l'organisation.

Si le projet de féminisation des métiers de la Construction peut s'appréhender comme un projet innovant, il doit avant tout s'inscrire dans une dimension stratégique globale. Pour l'entreprise CARI, l'intégration des femmes a été conçue comme un moyen de recrutement permettant d'élargir les publics cibles en matière de «sourcing ». Mais l'importance accordée au projet se double également d'une dimension stratégique forte basée sur la notion de changement, qui explique l'intégration du projet parmi les objectifs de la Direction générale. Au-delà de la féminisation en elle-même, c'est la capacité des équipes CARI à se remettre en 
question qui est recherchée. Le secteur de la Construction devient aujourd'hui de plus en plus complexe et, paradoxalement, repose encore sur un mode de fonctionnement classique, aux pratiques anciennes et sérieusement ancrées. Une partie de ces pratiques témoigne de la culture du métier et en constitue le cœur, mais le mécanisme général est bien souvent empreint également d'une inertie forte, inadaptée aujourd'hui dans un contexte changeant où, les normes, les conditions de travail, les lois sont de plus en plus exigeantes. De ce fait, l'arrivée des femmes représente une manière d'induire un changement des comportements et d'apprendre à concevoir différemment la réalité, au-delà des habitudes, au-delà des représentations collectives, et d'être à terme, soi-même porteur de changement. La féminisation est donc appréhendée par l'entreprise CARI comme un gage d'amélioration de la performance en développant la capacité à se remettre en cause et à s'adapter à de nouveaux contextes.

L'expérience menée au sein de l'entreprise a permis la réappropriation de méthodes d'intégration et de suivi renforcées par l'organisation. Le projet a nécessité de mettre en place un partenariat fort avec les différents acteurs de l'emploi, appréhendés ici dans un spectre très large afin d'identifier un maximum de sources de candidatures. Ce challenge entrepris a permis de faire reconnaître l'entreprise comme une structure à même de déployer des dispositifs innovants et de nouer des liens forts compte tenu de l'envergure du projet. Dans le contexte actuel, une dimension importante des problématiques de recrutement devient la fidélisation des nouvelles candidatures. A posteriori, il a été remarqué que les outils mis en place pour préparer et faciliter l'accueil des femmes pouvaient s'avérer utilisables pour l'intégration de l'ensemble des candidats du fait des résultats obtenus. Les recherches et les remises en cause induites par le projet bénéficient ainsi à l'ensemble des salariés en impactant sur la politique et les outils de gestion des ressources humaines.

D'autres effets ont été identifiés, au vu des résultats obtenus lors de l'intégration de groupes féminins dans l'industrie. Ils sont aujourd'hui en cours d'évaluation chez CARI :

- L'arrivée des femmes a modifié les règles aussi bien au niveau du collectif que des relations individuelles. La mixité semble en effet avoir induit une certaine forme de respect plus forte liée à la présence des femmes. Certaines stagiaires en ont d'ailleurs elles-mêmes témoigné mais cela n'a pas encore été vérifié sur l'ensemble des équipes.

- Dans les équipes masculines où des femmes ont été intégrées, les résultats en matière de sécurité se sont améliorés du fait d'un meilleur suivi des règles de sécurité : cet axiome est important dans la mise en œuvre de ce projet compte tenu de la fréquence et de la gravité des accidents du travail dans le secteur du BTP.

- L'arrivée des femmes semble être aussi vecteur d'une amélioration de la productivité, même si certains hommes la considèrent comme une remise en cause du monopole masculin. On identifie également le désir de 
constituer un modèle aux yeux de personnes étrangères au groupe afin de montrer une image positive de son travail.

\section{Conclusion}

Un groupe à dominante culturelle masculine qui prouve sa capacité à accueillir des femmes dans ses équipes, peut modifier sérieusement l'image du secteur. Il s'agit dans un premier temps d'une modification de la perception des mentalités : le groupe est ouvert et a la capacité de se remettre en cause. C'est un vecteur important pour l'image du métier, L'intégration d'équipes féminines, enfin, dans les métiers du BTP peut également modifier la perception de la pénibilité de ces métiers, encore fortement reliée dans les représentations collectives à la notion de force physique.

La conclusion la plus forte du projet se trouve d'ailleurs ici : il ne s'agit pas finalement de dévoiler le fait que les femmes sont capables de travailler dans les métiers du BTP ou de développer des stratégies d'adaptation dans des groupes masculins. Cette expérience souligne que, à l'encontre des hypothèses de départ, les résistances masculines au changement sont sans doute importantes, mais que les hommes disposent également d'un ressort pour modifier leurs attitudes, leurs habitudes et intégrer au sein de leur groupe constitué, des femmes, acceptant par là même d'initier un nouveau modèle d'organisation.

\section{Bibliographie}

- Conseil Economique et Social (2007), «La place des femmes dans les lieux de décision : promouvoir la mixité », Rapport $\mathrm{n}^{\circ} 6$

- A.F. Bender, (2004), «Egalité Professionnelle ou gestion de la diversité : quels enjeux pour l'égalité des chances », Revue Française de Gestion, $N^{\circ} 151$.

- A. F. Bender, F. Pigeyre (2003), «Gestion des ressources humaines et diversité : les cadres et les ingénieur(e)s au regard du genre ». Journée de recherche GDR Cadres. Mage. IRESCO. Paris.

- A. Boyer, M.J Scotto (2006), «La mixité du genre est-elle un nouvel enjeu managérial dans l'entreprise française ? » Richesse de la diversité. Actes des 2ème rencontres internationales de la diversité. IAE Corte.

- P. Bourdieu (2002) La domination Masculine, Collection Points Essai, Seuil.

- F. Brillet, (2007), «Vers la parité professionnelle dans les entreprises », Personnel, $\mathrm{N}^{\circ} 477$, pp. 23-26. 
- F Bry, J Ballet (2006), «Responsabilité sociale de l'entreprise : la mixité femmes/hommes et le plafond de verre ». La lettre du Management Responsable, Esdes, pp. 23-38, mai.

- M. Capron, F Quairel-Lanoizelée, (2004) Mythes et réalités de l'entreprise responsable, La Découverte.

- A. Cornet (2002), «Gendermainstreaming et approche différenciée selon les sexes: quel impact pour la gestion?» Management international, $\mathrm{N}^{\circ} 7.1$, pp. 1-10, Montréal.

- M. Cornut-Janin (2000) Féminin et Féminité. 2ème édition. PUF.

- T. Cox (1991), «The Multicultural Organization», Academy of Management Executive. vol.5, pp. 34-45.

- C. Derrez (2007), «BTP : où sont les femmes ? », www. spécial. sudouest. com.

- G. Duby, M. Perrot, F. Farge (1992) Histoire des femmes, Tome 5, Plon.

- EMSIS (2004), «Femmes dans le Bâtiment: l'image et les représentations sociales du bâtiment », Etude réalisée dans le cadre du protocole d'accord du 2 février 2002 en faveur de l'accès des femmes aux métiers du bâtiment.

- S. Fortino (2002) La mixité au travail, La Dispute.

- M. Godelier (2003) La production des grands hommes. Pouvoir et domination masculine chez les Baruya de Nouvelle Guinée, Flammarion.

- N. Gorbatko, A.M. Gelonek (2007), «Femmes : Inégalité puissance 10 ». TGV Magazine, janvier.

- INSEE (2004) Femmes/Hommes. Regards sur la Parité.

- Y. Kergoren (de) (2007), «Les femmes sont encore trop rares dans la hiérarchie », Rubrique Organisation, La Tribune, 20 octobre.

- Livre vert de la Commission Européenne (2001).

- C. Montaigne (2007), « Ces derniers bastions masculins qui tombent ». Management. Les Echos, 8 mars.

- J.M. Peretti (2006), «Richesse de la diversité dans l'entreprise ». Richesse de la Diversité, Collection Entreprendre, Vuibert.

- P. Prasad, A. Prasad, J.M. Cavanaugh (2002), "Designing difference : management and the discourse of fashion". Colloque international EGOS, Barcelone, juillet.

- D. Perrotte (2007), « Deux femmes cadres sur trois se disent victimes de discrimination professionnelle », Les Echos, 8 février.

- A.Pétrovic (2006), «Les femmes cherchent toujours leur place au plus haut niveau » La Tribune, 27 septembre. 
- A.Rapin (1999), «Entretien avec Michelle Perotte», Label France, p.37, octobre.

- S. Regy (2007,) «Les femmes arrivent peu à peu sur les chantiers », Le Moniteur, 16mars.

- D.Sabbagh, (2004), «La tentation de l'opacité », La discrimination positive, pp. 5-18, Seuil

- D.Thomas, R. Ely (1996), «Making differences matter: a new paradigm for managing diversity", Havard Business Review, 09/10.

- D.Weltzer-Lang (2002), (sous la direction de) «Les hommes en changement » Actes du séminaire européen sur les résistances masculines, p 192, Université de Toulouse le Mirail.

- R.K. Yin (1994) Case study research, design and methods, 2ème Edition, Sage Publication.

\section{Notes}

$[1]$

Etude ORSE (2004). L'accès des femmes aux postes de décision des entreprises, p. 59.

[2]

La promotion des femmes aux postes de décision. 199. BIT

[3]

Kandola, R. Fullerton, J. (1994). Diversity: more than just an empty slogan. Personnel Management.

$[4]$

Boyer A, Scotto M.J. (2006). La mixité du genre est elle un nouvel enjeu managérial dans l'entreprise française. Richesse de la diversité. Actes des 2ème rencontres internationales de la diversité. IAE Corte.

[5]

Commission Green Paper 2001. «Promoting a European Framwork for Corporate Social Responsability »

[6]

Commission Green Paper 2001. «Promoting a European Framwork for Corporate Social Responsability »

[7]

Pétrovic, A.( 2006). Hommes et Expertise. La Tribune. 27 Septembre.

[8]

Pétrovic, A.( 2006). Hommes et Expertise. La Tribune. 27 Septembre.

[9]

de Kergoren, Y. 2006. Organisation. La Tribune. 20 octobre.

$[10]$

Brillet, F. (2007). Management. Personnel. Février/mars

[11]

Perrote, D. (2007). Social. Les Echos. 8 février. 


\section{$[12]$}

Launay, P. (2007). Dépêche l'AEF (Agence Education, Emploi Formation) du 8 mars. $[13]$

Le Label Egalité a été mis en place fin 2004, à l'initiative du Ministère délégué à la cohésion sociale et à la parité. Il récompense l'exemplarité des engagements des entreprises, administrations ou associations dont le dossier a été jugé recevable par l'AFAQ, organisme de certification.

L'évaluation se fait sur plusieurs critères répartis dans 3 champs : les actions menées dans l'entreprise en faveur de l'égalité professionnelle, la gestion des ressources humaines et du management et enfin la prise en compte de la parentalité dans le cadre professionnel. Encouragé par l'Etat, soutenu par les partenaires sociaux, il peut être décerné à toute entreprise, quelle que soit sa taille ou son activité (une version à destination des PME-PMI existe depuis 2006). Source : Site du Ministère délégué à la cohésion sociale et à la parité.

$[14]$

Orse (2004). Répertoire des accords d'égalité professionnelle.

\section{[15]}

Roumier, A. (2007) Entretien du 12 février.

$[16]$

Yin (1994) définit six sources de données mobilisables dans le cadre d'une étude de cas : la documentation, les archives, l'entretien, l'observation directe, l'observation participante, et la simulation.

[17]

Paul Leroy-Beaulieu (1843 -1916), économiste français.

\section{[18]}

Maurice Godelier cité par Marie Durut Bellat. 1995. Garçon et filles à l'école de la différence. Ephesia. La Découverte.

\section{[19]}

INSEE (2003). Enquête Emploi.

\section{[20]}

Reiss.C (2007). AEF. Dépèche 73483.

[21]

Le BTP au féminin (2007). www. batiactu. com

[22]

Cité par Montaigne. C. (2007). Ces derniers bastions masculins qui tombent. Les Echos. 8 mars.

[23]

Montaigne. C. (2007). Ces derniers bastions masculins qui tombent. Les Echos. 8 mars.

\section{[24]}

Regy.S.( 2007). www. lemoniteur-expert. com

[25]

Le BTP au féminin (2007). www. batiactu. com

[26]

L'homme de Marbre. 1976. Film de Andrzej Wajda.

[27] 
Emsis (2004). L'image et les représentations sociales du batiment, p. 9.

\section{[28]}

Etude menée en 2005 par le Centre régional d'orientation professionnelle de l'AFPA Aquitaine, le laboratoire de psychologie sociale des insertions de l'université de Bordeaux 2 et l'Union nationale des associations Retravailler.

[29]

Baron F. (2006). Métiers atypiques. AEF. Dépêche 62429.

\section{[30]}

Ce label est obtenu après dépôt d'un dossier de candidature auprès de l'AFAQ Afnor Certification. Il est délivré par une commission composée de membres des organisations patronales et syndicales représentatives ainsi que de représentants de l'Etat. Il est attribué pour une durée de 3 ans, renouvelable.

[31]

CARI est fusion d'entreprises régionales dont les spécificités sont multiples : la plupart était des entreprises familiales, anciennes, avec un ancrage territorial fort et un principe de management de proximité. Les deux principales entreprises du groupe ont plus de cent ans aujourd'hui. De ce fait, dans l'état actuel des connaissances, il n'est pas possible d'affirmer ici que ces entreprises n'ont pas, à un moment donné, participé aux grandes vagues de migration, notamment dans les années 70. Il s'agit bien entendu ici d'une hypothèse.

[32]

Durant cette même période où le projet de féminisation a été mis en œuvre, CARI a développé un projet à titre expérimental afin de recruter des ouvriers au Portugal, en développant un mécanisme d'intégration responsable et un suivi rapproché. Cette expérience nous a confortés dans les choix que nous avions opérés. En effet, le temps consacré au déploiement de ce projet, au vue des résultats obtenus (niveau de qualification moyen des personnes recrutées, difficulté d'intégration au sein des équipes et du territoire, et enfin impossibilité d'envisager une intégration pérenne via un CDI) ont été mis en perspective des projets d'insertion menés actuellement au sein de l'entreprise et dont les résultats sont aujourd'hui bien meilleurs.

\section{[33]}

Il est important de mentionner que les représentants du personnel, lorsqu'ils ont été consultés, n'ont émis aucune remarque sur la production de ce rapport et les résultats et les enseignements que l'on peut en tirer n'ont été à l'origine d'aucune réclamation de leur part.

\section{[34]}

Il s'agit là d'une obligation légale dont le coût est, somme toute, très relatif. Par ailleurs, l'arrivée de très nombreux stagiaires en cours de formation, au même moment a constitué également un frein car il est demandé à chaque chantier, en plus de la réalisation de chaque ouvrage, de travailler à la formation de personnes qui ne sont pas compétentes immédiatement et dont pourtant la qualification est aujourd'hui nécessaire pour assurer les effectifs des équipes de demain. Si jadis, ce travail de transmission se faisait spontanément, cette attitude s'est aujourd'hui un peu perdue. Cependant, la facturation des jeunes stagiaires, y compris des femmes, a été sortie de la comptabilité des chantiers pour éviter que cela ne décourage l'accueil de ces nouveaux et ces nouvelles candidates. 


\section{$[35]$}

Il convient de nuancer ce propos : en effet, dans le cadre de la revalorisation des métiers du BTP, un projet se structure chez CARI afin de sensibiliser l'Education Nationale et les jeunes scolarisés en collèges et lycées notamment à l'intérêt du secteur. Stages en entreprise, stage de découverte des métiers, option Découverte des Métiers, forum et intervention dans les écoles, accueil et journée de sensibilisation à destination des professeurs et proviseurs, travail sur le rapprochement entre entreprise et éducation nationale via les rectorats, ... les initiatives sont nombreuses et c'est l'occasion pour l'entreprise, de largement communiquer sur ce projet de féminisation de nos filières, bien en amont. Dans le cadre de la Journée Internationale des femmes du 8 mars 2007, certaines ouvrières de CARI ont témoigné directement dans des écoles. Enfin, à titre anecdotique, une maçonne qui travaillait sur un chantier de rénovation au sein d'un lycée a également témoigné des réactions des élèves, de leur surprise de voir une femme sur un chantier et notamment des longues discussions avec le public féminin pour les encourager et les motiver en direct, à s'orienter vers la Construction...

\section{[36]}

Le projet s'organisait autour d'une période d'environ 9 mois en contrat de professionnalisation, période durant laquelle la stagiaire est intérimaire chez ADIA et qui se termine par une embauche en CDI chez CARI. L'intervention d'une personne de chez ADIA, l'employeur légal de la stagiaire durant sa formation, est importante car elle est située en dehors du chantier et de CARI et permet donc une liberté de parole plus grande de la part de la stagiaire.

\section{[37]}

Un des problèmes rencontrés et récurrents a porté sur la médiatisation du projet : il a été décidé d'accorder une place importante à ce projet en terme de communication, pour attirer des candidatures féminines mais aussi pour témoigner de l'ouverture de l'entreprise et du caractère innovant du projet. Les médias qui sont intervenus ont presque systématiquement émis le souhait de rencontrer des stagiaires et bien souvent cela a constitué un facteur nuisible à l'intégration : en effet, l'attention apportée à ces stagiaires par les journalistes, et qui jusque là n'avait jamais été apporté au reste de l'équipe, a suscité des reprises de réactions hostiles visant directement les candidates. De ce fait, les actions de communication sur le projet ont fait l'objet d'un encadrement spécifique et limité dans le temps. 